\title{
The influence of thermal fluctuations on Coulomb blockade edge in small Josephson junctions with linear growing of voltage
}

\author{
I.N. Askerzade \\ Department of Computer Engineering and Center of Excellence of Superconductivity Research, Ankara University \\ Ankara, TR06100, Turkey
}

Institute of Physics Azerbaijan National Academy of Sciences 33, H. Cavid 33, Baku, AZ1143, Azerbaijan

E-mail: imasker@eng.ankara.edu.tr

Received October 3, 2017, published online January 25, 2018

\begin{abstract}
In this study we carried out the analysis of the thermal fluctuations on Coulomb blockade edge in small Josephson junctions with linear growing of voltage. It was shown that, charge fluctuation is determined by the energy ratio-parameter of small size Josephson junction, temperature and growing rate of voltage.
\end{abstract}

PACS: 73.23.Hk Coulomb blockade; single-electron tunneling;

74.81.Fa Josephson junction arrays and wire networks.

Keywords: Josephson junction, Coulomb blockade, thermal fluctuations.

\section{Introduction}

Recent years the study of Josephson junctions with small capacitance becomes very attractive. The properties of small tunnel junctions are influenced by the external enviroment, i.e., by external leads attached to the junction. In small junctions Coulomb blockade arises of Cooper pairs, which means that such junctions can transfer individual superconducting pairs and generate Bloch oscillation [1-3]. Coulomb blockade appears when

$$
\min \left\{\hbar \omega_{p}, \hbar \omega_{c}\right\}>E_{J},
$$

where $\omega_{p}=\left(2 e I_{c} / \hbar C\right)^{1 / 2}$ is the plasma frequency, $\omega_{c}=2 e I_{C} R_{N} / \hbar$ is the characteristic frequency of Josephson junction with critical current $I_{c}$, normal resistance $R_{N}$ and capacitance $C$ and Josephson energy $E_{J}=\hbar I_{C} / 2 e$. In terms of resistance, Equation (1) can be rewritten as

$$
R_{N}>R_{Q}=\frac{\hbar}{4 e^{2}}
$$

where $R_{Q}=1 \mathrm{k} \Omega$ is the quantum unit of resistance.

The important pecularity of these junctions is the nonlinear differential capacitance $C_{B}$,

$$
C_{B}(q)=\left(\frac{d^{2} E_{0}(q)}{d q^{2}}\right)^{-1},
$$

which is related with the dispersion relation $E_{0}(q)$ of energy lower band [1-3] and $q$ is the quasicharge $(-e<q<e)$. For the describing dynamics of quasicharge in small Josephson junctions, we should take into account Bloch inductance $L_{B}(q)$ also [4]. The corresponding equation for quasicharge $q$ takes a form [4]

$$
L_{B}(q) \frac{d^{2} q}{d t^{2}}+R_{N} \frac{d q}{d t}+V(q)=V_{e},
$$

where $V_{e}$ is voltage in circuit, $L_{B}(q)$ is the Bloch inductance, $R_{N}$ is the resistance of Josephson junction. Periodical function $V(q)$ in Eq. (4) replace term $\sin \varphi$ in equations for usual Josephson effect description [5,6]. Expression of $V(q)$ is presented below $(-e<q<e)$ and was used in [7-9]

$$
V(q)=\frac{e}{C} \frac{\frac{q}{e}-\left(\frac{q}{e}\right)^{3}}{\sqrt{\left(\left(\frac{q}{e}\right)^{2}-1\right)^{2}+\frac{\kappa^{2}}{4}}} .
$$

It is well know that small Josephson junctions are characterized by the ratio $\kappa=E_{J} / E_{C}$, where $E_{C}=e^{2} / 2 C$ is Coulomb energy, $C$ is capacity of junction [1-3]. The case of $\kappa>>1$ correspond to the usual Josephson effect and in this case can be neglected by the effects of correlation in Cooper pair tunneling. In the opposite case due to small 
capacity, Coulomb energy $E_{C}$ becomes considerably, which leads to Coulomb blockade under tunneling of Cooper pair [8,9].

Dynamical properties of small Josephson junctions partially was investigated in [9]. In $I V$ curve of small Josephson junctions, at voltages below Coulomb blockade edge $V_{0}=e / C$ average supercurrent is approximately zero [10]. This stage correspond to linear charging of electrodes [7-9]. When charge in capacitor approachs the level $e$, the supercurrent becomes nonzero and as result the electrodes exchange a Cooper pair. In the presence of thermal fluctuations, take place effect of fluctuations of Coulomb blockade edge in $I V$ curve. Detail analysis of similar behavior was not conducted yet. In this study we develop theory for the estimation thermal fluctuations of Coulomb blockade edge of small Josephson junctions taking into account Bloch inductance (see Eq. (4)). In consideration of small Josephson junction with bias, we present dynamics of junction with linear growing voltage.

\section{Basic equations}

As shown in [1,2,9], the dynamical behavior of a small junction is similar to the behavior of a particle in the potential field of a form

$$
U(q)=E_{0}(q)-V_{e} q
$$

When the voltage across junction rises slowly, the energy barrier $U(q)=E_{0}(q)-V_{e} q$ gradually lowers and thermal fluctuations may initiate Cooper pair tunneling. For the probability of the tunneling on a time $t$, we can write $[5,6]$

$$
Q(t)=1-\exp \left[-\int_{0}^{t} \Gamma(q(t)) d t\right]
$$

where tunneling rate $\Gamma(t)$ can be expressed as [11] (see also [12])

$$
\Gamma(t)=\frac{\omega_{A}}{2 \pi} \mathrm{e}^{-\frac{\Delta U}{k T}}
$$

In Eq. (8), $\omega_{A}$ is the frequency attempt in the minimum of the potential barrier and $\Delta U$ is the height of the barrier, i.e., $\Delta U=U\left(q_{\max }\right)-U\left(q_{\min }\right) ; q_{\max }$ and $q_{\min }$ correspond to the maximum and minimum of the function from the Eq. (6), respectively. Also in the case of linearly growing voltage across Josephson junction

$$
\begin{gathered}
U\left(q_{\max }\right)=E_{C}-\frac{E_{J}}{2}, \\
U\left(q_{\max }\right)=\frac{C V_{e}^{2}}{2} .
\end{gathered}
$$

Calculation similar to [6] and using (9a), (9b), leads to expression for the height potential barrier

$$
\Delta U=U\left(q_{\max }\right)-V_{e} q_{\max }+\frac{C V_{e}^{2}}{2} .
$$

For the calculation of probability of tunneling of Cooper pairs, we consider $\frac{V_{e}}{V_{C}}=\alpha t, V_{C}=e / C$, the case of linearly growing voltage across junction. Corresponding expression has a form

$$
Q(t)=1-\exp \left[-\frac{\omega_{B}}{2 \pi} e^{-\frac{1-\kappa / 2}{\gamma} t_{0}^{\prime}} e^{-\frac{\alpha^{2} t^{2}}{\gamma}+\frac{2 \alpha}{\gamma} t} d t\right]
$$

where $\omega_{B}=1 / \sqrt{L_{B} C_{B}}$ is the frequency determined by the Bloch inductance of small Josephson junction [4,7], $\gamma=k T / E_{C}$ is the intensity of thermal fluctuations in units $E_{C}$. Calculation leads to final result for tunneling probability

$Q(t)=1-\left[\exp -\frac{\omega_{B}}{4 \pi} \frac{\sqrt{\pi \gamma}}{\alpha} \mathrm{e}^{\frac{\kappa}{2 \gamma}}\left(\operatorname{erf} \frac{\alpha t^{\prime}-1}{\sqrt{\gamma}}+\operatorname{erf} \frac{1}{\sqrt{\gamma}}\right)\right]$,

where erf $(\ldots .$.$) is the error function.$

\section{Result and discussions}

Using Eq. (12) under condition $\gamma=k T / E_{C}<<1$ and $\alpha<\gamma$, we can estimate fluctuation of Coulomb blockade edge, as similar to the case of usual Josephson junction $(\delta V=\delta q / C)$

$$
\frac{\delta q}{e}=\frac{2 \pi \alpha}{\omega_{B}} \exp \left(-\frac{\kappa}{2 \gamma}\right)
$$

In estimation of Bloch frequency $\omega_{B}=1 / \sqrt{L_{B} C_{B}}$, we will use expression for Bloch inductance

$$
L_{B}(q) \approx \frac{\Phi_{0}}{2 \pi I_{C}}\left(1+\frac{1}{\sqrt{2 \kappa}}\right)
$$

from [7] and as result we have

$$
\omega_{B}=\frac{\omega_{J}}{\left(1+\frac{1}{\sqrt{2 \kappa}}\right)}
$$

Result of calculations using Eq. (13) presented in Fig. 1. As you can see from Fig. 1, increasing of charge blockade effects $\kappa=\frac{E_{J}}{E_{C}} \rightarrow 0$ and thermal fluctuations $\gamma$ leads to increasing of normalized fluctuations of Coulomb blockade edge $\frac{\delta q}{e} / \frac{2 \pi \alpha}{\omega_{B}}$. Such conclusion in agreement with calculations for usual Josephson junction, which were carried out in [13-17]. However, in contrast to these papers, dependence of fluctuation on intensity of thermal fluctuations $\gamma$ has an another character. Another interesting 


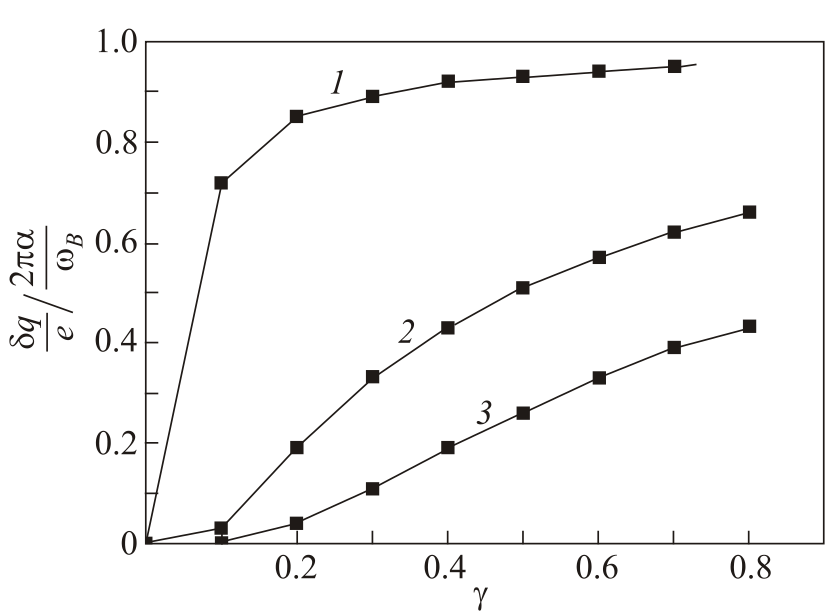

Fig. 1. Thermal fluctuations of Coulomb edge for different energy ratio parameter $\kappa=E_{J} / E_{C}$. $\kappa: 0.033$ (1), 0.333 (2), 0.666 (3).

result related with influence of a growing rate of voltage $\alpha$ on intensity of fluctuations $\delta q$. In the case of small junction with Coulomb blockade there is linear dependence of $\delta q$ on $\alpha$, while as shown in $[5,6,13,14]$, in the case of usual Josephson junction $(\kappa>>1)$ fluctuation of critical current $\delta i$ is proportional to $\left(\ln \frac{\gamma_{T}}{4 \pi \alpha^{\prime}}\right)^{2 / 3}$, where $\alpha^{\prime}$ is the growing rate of the current $i=\alpha^{\prime} t, \gamma_{T}=\frac{k T}{E_{J}}$. It means that, decreasing of $\alpha^{\prime}$ increase the fluctuation of current $\delta i$. It related with changing character of current of Cooper pairs, from inductive to the capacitive one (see Eq. (4)), as mentioned in [9].

For typical values of capacitance $C=0.1 f F$ and critical currents $I_{C}=0.1 \mu \mathrm{A}$ of small Josephson junctions, at which arises Coulomb blockade, voltage fluctuations can be estimated at the level $0.875 \mathrm{nV}$. In calculations energy ratio parameter $\kappa=E_{J} / E_{C}$ considered to be equal 0.333. Sampling rate of voltage taken for the typical case as $\alpha / \omega_{B} \sim 10^{-2}$. In estimations we use temperature $T \approx 0.16 \mathrm{~K}$ in similar to [10].

Thus, in this paper, the thermal fluctuations of Josephson junctions with a Coulomb blockade was investigated. Bloch oscillations in small Josephson junctions taken into account inclusion corresponding inductance in equivalent scheme. It was shown that the fluctuations of Coulomb blockade edge is determined by the parameters of Josephson junction. Estimation show that fluctuations of the Coulomb blockade edge can reach a level of $\mathrm{nV}$.

1. K.K. Likharev, A.B. Zorin, J. Low Temp. Phys. 59, 347 (1985)

2. D.V. Averin, A.B. Zorin, K.K. Likharev, Zh. Eksp. Teor. Fiz. 88, 692 (1985).

3. L.S. Kuzmin and D.B. Haviland, Phys. Rev. Lett. 67, 2890 (1991).

4. A.B. Zorin, Phys. Rev. Lett. 96, 167001 (2006).

5. I. Askerzade, A. Bozbey, and M. Canturk, Modern Aspects of Josephson Dynamics and Superconductivity Electronics, Springer, Springer (2017), p. 210.

6. K.K. Likharev, Introduction into Dynamics of Josephson's Junctions, Cordon Breach, New-York (1985).

7. I.N. Askerzade, Aut. Cont. Comp. Sci. 50, 10 (2016).

8. I.N. Askerzade and R. Samet, Tech. Phys. Lett. 34, 737 (2008)

9. I.N. Askerzade, Tech. Phys. 48, 1496 (2003).

10. L.S. Kuzmin, Yu.A. Pashkin, D.S. Golubev, and A.D. Zaikin, Phys. Rev. B 54, 10074 (1996).

11. H.A. Kramers, Physica 7, 284 (1940).

12. V. Ambegaokar and B.I. Halperin, Phys. Rev. Lett. 22, 1364 (1969).

13. O.V. Snigirev, Comm. Tech. Elect. 29, 2216 (1984).

14. I.N. Askerzade, Turkish J. Phys. 22, 811 (1998).

15. I.N. Askerzade, Tech. Phys. 43, 1123 (1998).

16. I.N. Askerzade, Tech. Phys. 45, 66 (2000).

17. I.N. Askerzade, Tech. Phys. 51, 393 (2006). 